\title{
Level of depression and hopelessness among women with infertility during the outbreak of COVID-19: a cross-sectional investigation
}

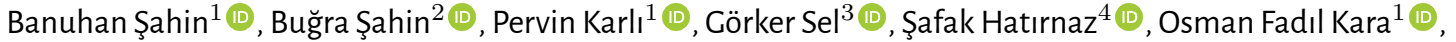 \\ Andrea Tinelli ${ }^{5,6, * \text { (D) }}$ \\ ${ }^{1}$ Department of Obstetrics and Gynecology, Amasya University Sabuncuoglu Serefeddin Training and Research Hospital, 05100 Amasya, Turkey \\ ${ }^{2}$ Clinics of Cynecology and Obstetrics, Turhal State Hospital, 60300 Tokat, Turkey \\ ${ }^{3}$ Department of Obstetrics and Cynecology, Faculty of Medicine, Zonguldak Bülent Ecevit University, 67100 Zonguldak, Turkey \\ ${ }^{4}$ Department of Cynecology and Obstetrics, IVFUnit, Medicana International Hospital, 06520 Samsun, Turkey \\ ${ }^{5}$ Department of Obstetrics and Cynecology, "Verisdelli Ponti" Hospital, Scorrano, 73100 Lecce, Italy \\ ${ }^{6}$ PhystechBioMed School, Faculty of Biological \& Medical Physics, Moscow Institute of Physics and Technology (State University), Dolgoprudny, 141701 \\ Moscow Region, Russia \\ *Correspondence: andreatinelli@gmail.com (Andrea Tinelli)
}

DOI:10.31083/j.ceog.2021.03.2435

This is an open access article under the CC BY 4.0 license (https://creativecommons.org/licenses/by/4.0/).

Submitted: 25 December 2020 Revised: 28 January 2021 Accepted: 8 February 2021 Published: 15 June 2021

Background: Novel coronavirus disease 2019 (COVID-19) outbreak negatively impacted on the mental wellbeing of infertile couples, because of the World Health Organization's recommendation to stop all fertility treatments. This study aims to investigate the level of depression and hopelessness during the COVID-19 outbreak, in women with primary and secondary infertility. Methods: A 16-item online questionnaire was applied to a total of 220 women with primary and secondary infertility in June 2020. Participants' knowledge and concerns about COVID-19 pandemic stop of treatments and follow-up preferences were evaluated. In addition, the Beck Depression Inventory $(\mathrm{BDI})$ and the Beck Hopelessness Scale $(\mathrm{BHS})$ were adapted to Turkish and applied to each participant. Total scores of inventories between women were calculated. The correlation between depression and hopelessness levels of the groups was evaluated. Results: The BDI and BHS mean scores of 220 infertile women who completed our questionnaire were $14.63 \pm 9.123$ and $5.43 \pm 5.026$ and Cronbach's $\alpha$-values were 0.853 and 0.904 , respectively. It was found that 142 women with secondary infertility had higher mean depression and hopelessness scores $(15.92 \pm 9.758,11.81 \pm 6.501 ; P=0.001)$ VS 78 women with primary infertility $(6.51 \pm 5.262,3.78 \pm 3.670 ; P=$ $0.000)$. A strong positive correlation was detected between depression and hopelessness levels for each group $(r=0.625, P=0.000$ and $r=0.740, P=0.000$ ). Conclusions: Total depression and hopelessness scores of both groups were in a strong positive correlation with primary and secondary infertility during the COVID-19 outbreak. Particularly, women with secondary infertility had mild depression and low levels of hopelessness.

\section{Keywords}

COVID-19 outbreak; Infertility; Assisted reproductive technology; Depression; Hopelessness

\section{Introduction}

The World Health Organization (WHO) declared that novel coronavirus disease 2019 (COVID-19) became a global pandemic on 11 March 2020 [1]. Since then, several guidelines, epidemiological bulletins and letters of recommendations have been published and updated in the medical field. In reproductive medicine, the European Society of Human Reproduction and Embryology (ESHRE) and the American Society for Reproductive Medicine (ASRM) suggested that patients with infertility should consider putting off their pregnancy plans [2, 3]. All assisted reproductive technology (ART) treatments, including ovulation induction, intrauterine insemination (IUI), in vitro fertilization (IVF), oocyte and sperm cryopreservation, as well as fresh or frozen embryo transfer, were postponed by the fertility societies globally.

These decisions were made for possible complications of ART and pregnancy, mitigating the unknown risk of maternal fetal transmission in COVID-19-positive mothers and supporting the necessary reallocation of healthcare resources [4]. All societies advocated that initiated cycles were to be completed and a freeze-all policy adopted, whereas transfers in preparation were discussed and, mostly, were postponed [5]. Subsequently, ESHRE published a document on restarting infertility treatment during the COVID-19 pandemic, advising high-risk patients (diabetes mellitus, hypertension, those on immune suppressant therapy, past transplant patients and those with lung, liver or renal disease) to stop their ART treatment, finally recommending to all patients to postpone their treatment [6].

Conservative measures on IVF cycles stopping cycles during COVID-19 outbreak aggravate patients' anxiety and the 
fear of remaining infertile for the possibility of losing the chance of fertilization. Generally, infertility is known to be a major cause of stress, as infertile couples were reported to have higher levels of anxiety and depression. Managing these patients by alleviating their symptoms is highly recommended during their fertility challenge [7].

Because of this pandemic problem that recently arose, surveys are needed to develop evidence-based strategies to reduce the negative psychological effects and psychiatric symptoms during the COVID-19 outbreak. In fact, studies have been administrated to general population, medical students, children, healthcare workers and COVID-19-positive patient groups [8-11].

Starting from these clinical findings, authors examined the mental health impacts of fertility treatment suspensions during COVID-19 pandemic by a cross-sectional investigation. This study aimed to measure the depression and hopelessness levels in women with primary and secondary infertility submitted to ART treatment during the COVID-19 outbreak, by an analysis of their thoughts, worries and expectations about discontinuing ART treatments. Authors divided infertility into primary and secondary for the psycho-attitudinal differences between couples who have never had pregnancies and those who have had pregnancies. The patients with primary infertility having never had pregnancies in the past, were logically more charged with expectations and hopes, unlike the latter.

\section{Materials and methods}

As the Turkish Government suggested that citizens should minimize face-to-face interactions, potential participants were invited to take part electronically by the providers of the current research. This research was designed to measure depression and hopelessness levels in infertile women undergoing or planning to undergo infertility treatment during the COVID-19 outbreak. An online survey platform, Google Form, was used to deliver the survey during 1-30 June 2020 period. A total of 220 women with primary and secondary infertility and aged 18-48 years voluntarily completed the on-line questionnaire. They were selected randomly via computer system. Patients were approached from the hospital database of infertility clinics in Mid-black sea region of Turkey. Verbal informed consents were recorded at the beginning of the interviews of participants, via telephone. The power analysis was performed based on an expected prevalence of major depression as $17 \%$ in infertile women [12]. The calculation showed that at least 217 participants would need to be enrolled with an alpha error level of 0.05 and a power of $80 \%$. The study was performed in accordance with the Declaration of Helsinki. Ethical approval for the study was granted by Amasya University's ethics committee (Decision no: 2020/6-36).

The inclusion criteria were: at least a primary school graduate, receiving infertility treatment (primary/secondary infertility), not diagnosed as having any psychiatric disorder, not using psychotropic medicine and agree to participate in the questionnaire and not abandon the survey during the questionnaire replies.

The structured questionnaire, inspired by previous research on the psychological effects of severe acute respiratory syndrome (SARS) outbreaks in Hong Kong [13], consisted of questions covering several areas: (1) socio-demographic and infertility features; (2) knowledge and concerns about COVID-19 ('Worries about being infected by COVID-19' Yes/No, 'Worries about infecting the baby by COVID-19' -No transmission/Ongoing/Termination); (3) thoughts on cessation of ART treatments during the COVID-19 pandemic ('Thoughts about stopping infertility treatments during COVID-19' -Yes/No, 'Thoughts about affecting the infertility treatment by COVID-19' -Yes/No); (4) responses to the Beck Depression Inventory (BDI) and Beck Hopelessness Scale (BHS). An online orientation session was applied prior to the survey for women to improve their understanding of various elements of BDI and BHS. Participants agreed to "not abandon the survey during the questionnaire replies".

The BDI was developed by Beck in 1961 to assess the risk of depression and severity of depressive symptoms [14]. The study subjects completed a questionnaire consisting of 21 questions estimating the severity of various depressive symptoms on a Likert scale of 0-3 (0, least severe; 3, most severe). Scores of 0-9 indicate no depression, 10-18 mild depression, 19-29 moderate depression and $>29$ severe depression. Adaptation of the BDI to Turkish and studies of reliability and validity were conducted by Hisli [15]. Cronbach's $\alpha$ coefficient for reliability of the Turkish version was reported as 0.80 [15].

The BHS was developed by Beck in 1974 to measure the future expectations of adults, consisting of 20 statements with a score range of 0-20 [16]. There are three subscales: emotions and expectations; motivation loss; and hope. Questions $1,6,13,15$ and 19 relate to emotions and expectations, questions $2,3,9,11,12,16,17$ and 20 relate to motivation loss and questions $4,7,8,14$ and 18 relate to hope. The individual is expected to mark 'right' for the statements they consider applicable to themselves and 'wrong' for those they consider inapplicable. The BHS is scored '1' for a suitable answer and '0' for an unsuitable answer. Scores of 0-3 indicate no or minimal hopelessness whereas scores of 4-8, 9-14 and $\geq 15$ indicate low, moderate and high levels of hopelessness, respectively. Adaptation of the BHS to Turkish and studies of reliability and validity were conducted by Durak [17]. Cronbach's $\alpha$-coefficient for reliability of the Turkish version was reported as 0.72 [17].

\subsection{Statistical analysis}

The study data were analysed statistically using IBM SPSS Statistics 21.0 (IBM Corp., Armonk, NY, USA). Descriptive statistics (mean, median, standard deviation, minimum, maximum, number, percentage) were used for data analysis. The distribution of the data was evaluated with the KolmogorovSmirnov test. For non-normally distributed parameters, the 
Mann-Whitney U test, the Kruskal-Wallis test, and Fischer's exact test were used for continuous variables and the chisquare test was used for categorical variables. Correlations were assessed using Pearson's correlation coefficient, along with the related $P$ values. Values with test results of $P<0.05$ were statistically significant.

\section{Results}

A total of 220 infertile patients completed online survey: 78 had primary infertility and 142 had secondary infertility. They had a mean age of $30.66 \pm 6.064$ years, ranged between 18 and 48 years. One hundred and forty-five women received medical, IUI or IVF treatment, and seventy-five women went through the testing/diagnosis process, up just before COVID-19 pandemic.

Demographic and clinical characteristics of enrolled patients, during the COVID-19 pandemic, were the following: the mean duration of marriage was $6.15 \pm 3.480$ years, the mean duration of infertility was $4.16 \pm 2.842$ years, $44.1 \%$ of women were graduated at University and $12.3 \%$ were on leave because of the COVID-19 outbreak, 17.7\% were healthcare workers (including doctors, dentists, dental hygienists, midwives, nurses, health officers and medical secretaries) (Table 1).

Table 1. Demographic and clinical characteristics of infertile women during the COVID-19 outbreak.

\begin{tabular}{lc}
\hline Variable $(n=220)$ & \\
\hline Age (years) & $60.66 \pm 6.064$ \\
Duration of marriage (years) & $4.16 \pm 2.842$ \\
Duration of infertility (years) & $67(30.5)$ \\
Educational status & $56(25.5)$ \\
$\quad$ Primary and secondary education & $97(44.1)$ \\
$\quad$ High school & \\
$\quad$ University & $115(52.3)$ \\
Occupation & $32(14.5)$ \\
$\quad$ Unprofessional & $39(17.7)$ \\
$\quad$ Educational workers & $34(15.5)$ \\
Healthcare workers & \\
Other professions & $142(64.5)$ \\
Employment & $51(23.2)$ \\
$\quad$ Unemployed & $27(12.3)$ \\
Employed &
\end{tabular}

Variables presented as mean \pm standard deviation and number (\%).

IUI, intrauterine insemination; IVF, in vitro fertilization.

Mean BDI and BHS scores were $14.63 \pm 9.123$ and $5.43 \pm$ 5.026 , respectively, for all infertile women. Mean emotions and expectations, motivation loss and hope subscale scores of the BHS were $4.13 \pm 1.413,2.39 \pm 1.856$ and $2.32 \pm 1.189$, respectively (Table 2 ). Cronbach's $\alpha$-values were calculated as 0.853 for the BDI and 0.904 for the BHS.

A comparison of variables in women with primary and secondary infertility was presented in Table 3 . Women with
Table 2. Psychological characteristics of infertile women during the COVID-19 outbreak.

\begin{tabular}{lc}
\hline Variable $(n=220)$ & \\
\hline Total BDI score & $14.63 \pm 9.123$ \\
Total BHS score & $5.43 \pm 5.026$ \\
Emotions and expectations score & $4.13 \pm 1.413$ \\
Motivation loss score & $2.39 \pm 1.856$ \\
Hope score & $2.32 \pm 1.189$ \\
\hline
\end{tabular}

Variables presented as mean \pm standard deviation. BDI, Beck

Depression Inventory; BHS, Beck Hopelessness Scale.

secondary infertility were significantly older than those with primary infertility $(P=0.000)$. The duration of infertility for both groups was similar $(P=0.531)$. Differences were not detected in 'Thoughts about stopping infertility treatments during COVID-19', 'Worries about being infected by COVID-19', 'Worries about infecting the baby by COVID19 ' or 'Thoughts about affecting the infertility treatment by COVID-19' for both groups $(P=0.755, P=0.057, P=0.263$ and $P=0.051$, respectively).

Forty women (51.28\%) with primary infertility and 103 (72.53\%) with secondary infertility had clinically depressive symptoms, whereas 23 (29.48\%) with primary infertility and 95 (66.90\%) with secondary infertility had clinical hopelessness symptoms. Total BDI and BHS scores were significantly higher in women with secondary infertility $(P=0.001$ and $P$ $=0.000$, respectively). Furthermore, the BHS subscale scores were statistically lower in women with primary infertility $(P$ $=0.000, P=0.003$ and $P=0.000$, respectively) (Table 4).

Total depression and hopelessness scores of both groups were in a strong positive correlation with primary and secondary infertility during the COVID-19 outbreak $(r=0.625$ and $r=0.740$, respectively; $P=0.000$ ) (Table 5).

\section{Discussion}

The COVID-19 pandemic has shocked the social and scientific world, interrupting any human relationship or contact due to the risk of transmission of the virus to mankind. Due to the negative psychological impact of the COVID-19 pandemic on women in general and, above all, in women submitted to ART/IVF in the pandemic period, apart the ESHRE and the ASRM recommendations [2, 3], also the Canadian Fertility and Andrology Society, announced on March 2020 their recommendations to immediately suspend all in-person fertility treatments throughout Canada and the U.S. indefinitely due to the COVID-19 pandemic [18]. This caused a large worldwide psychological stress in patient to submit to ART/IVF.

Authors, therefore, evaluated the same kind of infertile patients, but in the Turkish population, showing that primary and secondary infertile women had mild depression and low hopelessness levels during the outbreak, and patients with secondary infertility had higher BDI and BHS scores, than women with primary infertility. Moreover, increased BHS scores were correlated with increased BDI scores for 
Table 3. Comparison of variables in women with primary and secondary infertility during the COVID-19 outbreak.

\begin{tabular}{|c|c|c|c|}
\hline \multirow{2}{*}{ Variables } & Primary infertility & Secondary infertility & \multirow[t]{2}{*}{$P$ value } \\
\hline & $(\mathrm{n}=78)$ & $(\mathrm{n}=142)$ & \\
\hline Age (years) & $27.97 \pm 4.674$ & $32.43 \pm 5.923$ & 0.000 \\
\hline Duration of infertility (years) & $4.49 \pm 3.099$ & $4.20 \pm 3.256$ & 0.531 \\
\hline \multicolumn{4}{|c|}{ Thoughts about stopping infertility treatments during COVID-19 pandemic } \\
\hline Yes & $34(43.6)$ & $65(45.8)$ & \multirow{2}{*}{0.755} \\
\hline No & $44(56.4)$ & $77(54.2)$ & \\
\hline \multicolumn{4}{|c|}{ Worries about being infected by COVID- 19} \\
\hline Yes & $55(70.5)$ & $116(81.7)$ & \multirow{2}{*}{0.057} \\
\hline No & $23(29.5)$ & $26(18.3)$ & \\
\hline \multicolumn{4}{|c|}{ Worries about infecting the baby with COVID- 19} \\
\hline No transmission & $14(17.9)$ & $18(12.7)$ & \multirow{3}{*}{0.263} \\
\hline Ongoing & $64(82.1)$ & $121(85.2)$ & \\
\hline Termination & 0 & $3(2.1)$ & \\
\hline \multicolumn{4}{|c|}{ Thoughts about affecting the infertility treatment by COVID- 19} \\
\hline Yes & $15(19.2)$ & $45(31.7)$ & \multirow{3}{*}{0.051} \\
\hline No & $33(42.3)$ & $40(28.2)$ & \\
\hline Undecided & $30(38.5)$ & $57(40.1)$ & \\
\hline
\end{tabular}

Variables presented as mean \pm standard deviation and number (\%); Mann-Whitney $\mathrm{U}$ test and Chi-squared test; $P<0.05$.

Table 4. Comparison of psychological variables in women with primary and secondary infertility during the COVID-19 outbreak.

\begin{tabular}{lccc}
\hline & Primary infertility & Secondary infertility & \multirow{2}{*}{$P$ value } \\
\cline { 2 - 3 } & $(\mathrm{n}=78)$ & $(\mathrm{n}=142)$ & \\
\hline Total BDI score & $11.81 \pm 6.501$ & $15.92 \pm 9.758$ & 0.001 \\
$\quad 38(48.7)$ & $39(27.5)$ & \\
None & $27(34.6)$ & $63(44.4)$ & \multirow{2}{*}{0.011} \\
Mild depression & $11(14.1)$ & $29(20.4)$ & \\
Moderate depression & $2(2.6)$ & $11(7.7)$ & 0.000 \\
Severe depression & $3.78 \pm 3.670$ & $6.51 \pm 5.262$ & \\
Total BHS score & $46(58.9)$ & $47(33)$ & 0.001 \\
None/minimal & $25(32.1)$ & $64(45.1)$ & \\
Low hopelessness & $5(6.4)$ & $14(9.9)$ & 0.000 \\
$\quad$ Moderate hopelessness & $2(2.6)$ & $17(12)$ & 0.003 \\
High hopelessness & $1.90 \pm 1.534$ & $2.66 \pm 1.964$ & 0.000 \\
Emotions and expectations score & $1.85 \pm 1.058$ & $2.58 \pm 1.181$ & \\
Motivation loss score & $3.86 \pm 1.556$ & $4.63 \pm 0.927$ & \\
Hope score &
\end{tabular}

Variables presented as mean \pm standard deviation and number (\%); Mann-Whitney $\mathrm{U}$ test and Chi-squared test; $P<$ 0.05 .

each infertility group and, clinically, half of patients with primary infertility and more than $72 \%$ with secondary infertility had clinically depressive symptoms, whereas less than $30 \%$ with primary infertility and more than $65 \%$ with secondary infertility had clinical hopelessness symptoms.

Moreover, authors showed that half of the women with primary infertility and the majority of those with secondary infertility experienced depression; criticizing these results, authors would have expected the opposite, given that the latter already have a previous pregnancy, while the others did not miss one and therefore the expectation should be higher. It may be that the blockade had upset couples already with a child, due to a sudden negative reshaping of their lives, unlike the first.
Comparing our data with current literature, Esposito et al. [19] evaluated the impact of the COVID-19 pandemic on infertile couples' emotions, anxiety and future plans, in an observational study, performed by Italian ART centers and online forums on infertile couples' candidate to ART and whose treatment was blocked due to the COVID-19 lockdown. The COVID-19 lockdown had a moderate/severe psychological impact on infertile patients, women were more emotionally distressed, anxious and depressed than men and the recommendation to stop ART program during COVID-19 generated higher distress levels in infertile couples. Lawson et al. [20] evaluated perceptions of delayed fertility care secondary to the COVID-19 pandemic by a cross-sectional anonymous survey of $n=787 / 2287$ patients on recommendations to de- 
Table 5. Correlation between total depression and total hopelessness scores in women with primary and secondary infertility during the COVID-19 outbreak.

\begin{tabular}{lllc}
\hline \multirow{2}{*}{ Total hopelessness score } & \multicolumn{2}{c}{ Total depression score } \\
\cline { 3 - 4 } & & \multicolumn{1}{c}{$r$} & \multicolumn{1}{c}{$P$} \\
\hline \multirow{2}{*}{ Primary infertility group } & 0.625 & 0.000 \\
& Secondary infertility group & 0.740 & 0.000 \\
\hline
\end{tabular}

Pearson's correlation test; $P<0.05$.

lay fertility treatments due to the COVID-19 pandemic. Most participants were distressed by the delay of treatments and supplemental education increased acceptance of recommendations but did not decrease distress. Boivin et al. [21] analyzed appraisals, coping strategies and emotional reactions of patients to COVID-19 fertility clinic closures, by a crosssectional design, comprising a mixed-methods, English language, anonymous, online survey posted from April 9 to 21 to social media. The study was completed by 446 infertile women and patients appraised fertility clinic closure as having potential for a more negative than positive impact on their lives, and to be very or extremely uncontrollable and stressful. Participants reported more negative than positive emotions and, almost all participants reported stress, worry and frustration at the situation, while some expressed anger and resentment at the unfairness of the situation. Gordon et al. [22] examined the psychological impact of fertility treatment suspensions resulting from the COVID-19 pandemic and to clarify psychosocial predictors of better or worse mental health in 92 women from Canada and the United States, by a battery of questionnaires assessing depressive symptoms, perceived mental health impact, and change in quality of life related to treatment suspensions. Fifty-two of respondents endorsed clinical levels of depressive symptoms and fertility treatment suspensions have had a considerable negative impact on women's mental health and quality of life.

Yilmaz and Kavak [23] determined a mean BDI score of $28 \pm 1.08$ for infertile women, indicating a moderate level of depression. They also determined that women had increased feelings of hopelessness with increased severity of depressive symptoms. Yoldemir et al. [24] compared the depression between primary and secondary infertile couples and found mild depression was higher in the primary infertile women. In authors' investigation, the prevalence of depression was lower compared to this study, which consisted of infertile women who were mainly housewives. The infertile population in this study consisted mainly of women with university education, and having a job increases an individual's selfesteem and ability to cope with anxiety and depression. Depression not only affects the life of infertile individuals, but also impacts their treatment and follow-up [25].

Keskin et al. [26] highlighted hopelessness as an important factor known to reduce the chance of success in infertility treatment, defining it as "having negative expectations about the future". Social pressure, the challenge of infertility treatment and the duration of the diagnostic processes add up to the advanced levels of hopelessness of these women, whereas a high level of hope is a pivotal factor for coping with infertility problems [27]. Study' results highlighted $41 \%$ of women with primary infertility and more than $65 \%$ with secondary infertility with clinical hopelessness symptoms; as for depression, the lack of family upheavals in primary infertility could be an important factor, against the necessary family closure with offspring for the secondary infertility patients, due to the COVID-19 outbreak.

Infertile women's BHS score was determined by Kargin et al. as $5.6 \pm 4.2$ (low level of hopelessness) and by Kaya et al. as $3.81 \pm 2.87$ (minimal hopelessness) [28, 29]. In the present study, the BHS score was $5.43 \pm 5.026$ (low level of hopelessness) and the score was higher in women with secondary rather than primary infertility. As a result, the low level of hopelessness in the present study can be explained by the fact that patients wish to start treatment with a positive outlook, despite the COVID-19 pandemic. In addition, having a younger cohort of infertile women and no extended treatment modalities allowed them to present low levels of hopelessness. Moreover, it was found that women with secondary infertility experienced more hopelessness compared to women with primary infertility. Authors supposed that women who had a pregnancy, but could not achieve a positive result, may feel hopelessness towards experiencing the same problem again. Increased marriage rate may cause these negative feelings, due to the social pressure and loss of social status in relation to the lack of motherhood. In addition to the psychological problems of infertile patients during the pandemic, literature also reports perinatal problems related to COVID-19 pandemic, as reports on infants born free of COVID-19 but also on adverse perinatal outcomes, such as premature rupture of membranes and preterm delivery $[30,31]$. The reports generally refer to COVID-19 infection in the third trimester and not in the initial stages of pregnancy [32]. Recently, Cavalcante et al. [33] presented a negative impact of COVID-19 in steroidogenesis (male and female), sperm quality and folliculogenesis. The majority of infertile patients worried about being infected by COVID-19 and infecting the baby with COVID-19, although they continued ART treatment during the pandemic.

Infertile patients were also anxious and scared about the possibility of further compromising their chances of pregnancy, in relation to increasing age and the worse ovarian reserve during the ongoing COVID-19 pandemic emergency and the current recommendations to suspend ART treat- 
ment. So, psychological support was recommended for infertile patients searching for pregnancy, in order to avoid any uncertainty over their infertile condition. It might negatively affect their future reproductive choices, otherwise resulting in an increased prevalence of treatment drop out. Telemedicine and psychological support have established a useful tool for ART specialists [34]. Several digital platforms have quickly spread, valuable in favouring communication and the relationship between fertility physicians and infertile patients.

The strengths of this study were the possibility to reach patients via internet. In this way, despite the least possible face-to-face contact situation, it provided more opportunity to reach more infertile women. The limitation of the research was that it does not have a longitudinal course. Answers to the $\mathrm{BDI}$ and $\mathrm{BHS}$ may change as ART treatment progresses and the condition of the COVID-19 outbreak worsens.

\section{Conclusions}

Depression and hopelessness of infertile women are at mild levels during the COVID-19 pandemic. Women with secondary infertility had higher BDI and BHS scores than women with primary infertility. The majority of infertile women wanted to proceed with ART treatment, despite their worry over COVID-19 infection in themselves and their babies. Suggestion: There is a need for clinical and psychological counselling services to be provided by a team of infertility specialists and psychologists online to ensure ART treatment of all infertile women during the COVID-19 pandemic.

\section{Author contributions}

BŞ and GS designed the research study. BŞ and PK performed the research. BŞ analyzed the data. BŞ, OFK, ŞH and AT wrote the manuscript. All authors contributed to editorial changes in the manuscript. All authors read and approved the final manuscript.

\section{Ethics approval and consent to participate}

All subjects gave their informed consent for inclusion before they participated in the study. The study was conducted in accordance with the Declaration of Helsinki, and the protocol was approved by the Ethics Committee of Amasya University (approval number: 2020/6-36).

\section{Acknowledgment}

We thanks to every participant to take their time to survey.

\section{Funding}

This research received no external funding.

\section{Conflict of interest}

The authors declare no conflict of interest.

\section{References}

[1] World Health Organization. Coronavirus disease (Covid-19) technical guidance: surveillance and case definitions. World Health Organization. 2020.

[2] American Society for Reproductive Medicine. Covid-19: suggestions on managing patients who are undergoing infertility therapy or desiring pregnancy. 2020. Available at: https://www.reproductivefacts.org/news-and-publications/ne ws-and-research/press-releases-and-bulletins/covid-19-sugge stions-on-managing-patients-who-are-undergoing-infertility- $\mathrm{t}$ herapy-or-desiring-pregnancy/ (Accessed: 12 March 2020).

[3] European Society for Human Reproduction and Embriology. Coronavirus Covid-19: ESHRE statement on pregnancy and conception. 2020. Available at: https://www.eshre.eu/Press-Room/ ESHRE-News (Accessed: 19 March 2020).

[4] American Society for Reproductive Medicine. Patient management and clinical recommendations during the coronavirus (Covid-19) pandemic. Update \#1 (March 30, 2020 through April 13, 2020). 2020. Available at: https://www.asrm.org/globalassets/asrm/asrm-content/n ews-and-publications/Covid-19/Covidtaskforceupdate1.pdf (Accessed: 30 March 2020).

[5] Souza MDCB, Nakagawa H, Taitson PF, Cordts EB, Antunes RA. Management of ART and Covid-19: infertility in Times of Pandemic. What now? JBRA Assisted Reproduction. 2020; 24: 231232.

[6] European Society for Human Reproduction and Embriology. ESHRE guidance on recommencing ART treatments. 2020. Available at: https://www.eshre.eu/-/media/sitecore-files/Gui delines/COVID19/ESHRE-Guidance-on-Recommencing-ART -treatments_update-04052020.pdf (Accessed: 5 May 2020).

[7] Domar AD. The relationship between stress and infertility. BodyMind Interaction in Psychiatry. 2018; 20: 41-47.

[8] Zhang Y, Ma ZF. Impact of the Covid-19 pandemic on mental health and quality of life among local residents in liaoning province, China: a cross-sectional study. International Journal of Environmental Research and Public Health. 2020; 17: 2381.

[9] Cao W, Fang Z, Hou G, Han M, Xu X, Dong J, et al. The psychological impact of the Covid-19 epidemic on college students in China. Psychiatry Research. 2020; 287: 112934.

[10] Al-Rabiaah A, Temsah M, Al-Eyadhy AA, Hasan GM, Al-Zamil F, Al-Subaie S, et al. Middle East Respiratory Syndrome-Corona Virus (MERS-CoV) associated stress among medical students at a university teaching hospital in Saudi Arabia. Journal of Infection and Public Health. 2020; 13: 687-691.

[11] Lai J, Ma S, Wang Y, Cai Z, Hu J, Wei N, et al. Factors associated with mental health outcomes among health care workers exposed to coronavirus disease 2019. JAMA Network Open. 2020; 3: e203976.

[12] Chen T, Chang S, Tsai C, Juang K. Prevalence of depressive and anxiety disorders in an assisted reproductive technique clinic. $\mathrm{Hu}$ man Reproduction. 2004; 19: 2313-2318.

[13] Leung GM, Ho L, Chan SKK, Ho S, Bacon-Shone J, Choy RYL, et al. Longitudinal assessment of community psychobehavioral responses during and after the 2003 outbreak of severe acute respiratory syndrome in Hong Kong. Clinical Infectious Diseases. 2005; 40: 1713-1720.

[14] Beck AT, Ward CH, Mendelson M, Mock J, Erbaugh J. An inventory for measuring depression. Archives of General Psychiatry. 1961; 4: 561-571.

[15] Hisli N. The validity and reliability of the Beck Depression Inventory for university students. The Journal of Psychology. 1989; 7: 3-13.

[16] Beck AT, Weissman A, Lester D, Trexler L. The measurement of pessimism: the hopelessness scale. Journal of Consulting and Clinical Psychology. 1974; 42: 861-865.

[17] Durak A. Beck Umutsuzluk Ölçeği (BUÖ) geçerlik ve güvenirlik çalışması. Türk Psikoloji Dergisi. 1994; 9: 1-11. (In Turkish)

[18] Canadian Fertility \& Andrology Society. CFAS communication on 
Covid-19. 2020. Available at: https://cfas.ca/CFAS_Communica tion_on_COVID-19.html\#march18 (Accessed: 18 March 2020).

[19] Esposito V, Rania E, Lico D, Pedri S, Fiorenza A, Strati MF, et al. Influence of Covid-19 pandemic on the psychological status of infertile couples. European Journal of Obstetrics \& Gynecology and Reproductive Biology. 2020; 253: 148-153.

[20] Lawson AK, McQueen DB, Swanson AC, Confino R, Feinberg EC, Pavone ME. Psychological distress and postponed fertility care during the Covid-19 pandemic. Journal of Assisted Reproduction and Genetics. 2021; 38: 333-341.

[21] Boivin J, Harrison C, Mathur R, Burns G, Pericleous-Smith A, Gameiro $S$. Patient experiences of fertility clinic closure during the Covid-19 pandemic: appraisals, coping and emotions. Human Reproduction. 2020; 35: 2556-2566.

[22] Gordon JL, Balsom AA. The psychological impact of fertility treatment suspensions during the Covid-19 pandemic. PLoS ONE. 2020; 15: e0239253.

[23] Yilmaz E, Kavak F. The effect of stigma on depression levels of Turkish women with infertility. Perspectives in Psychiatric Care. 2019; 55: 378-382.

[24] Yoldemir T, Yassa M, Atasayan K. Comparison of depression between primary and secondary infertile couples. Gynecological Endocrinology. 2020; 36: 1131-1135.

[25] Purewal S, Chapman SCE, van den Akker OBA. Depression and state anxiety scores during assisted reproductive treatment are associated with outcome: a meta-analysis. Reproductive BioMedicine Online. 2018; 36: 646-657.

[26] Keskin G, Babacan Gumus A. Infertility: an examination hopelessness perspective. Journal of Psychiatric Nursing. 2014; 5: 9-16.
[27] Patel A, Sharma PSVN, Kumar P. "In cycles of dreams, despair, and desperation:" Research perspectives on infertility specific distress in patients undergoing fertility treatments. Journal of Human Reproductive Sciences. 2018; 11: 320.

[28] Kargın M, Ünal S. Determining hopelessness in infertile individuals. In New Symposium Journal. 2011; 49: 54-60.

[29] Kaya Z, Oskay U. Stigma, hopelessness and coping experiences of Turkish women with infertility. Journal of Reproductive and Infant Psychology. 2020; 38: 485-496.

[30] Schwartz DA. An analysis of 38 pregnant women with Covid-19, their newborn infants, and maternal-fetal transmission of SARSCoV-2: maternal coronavirus infections and pregnancy outcomes. Archives of Pathology \& Laboratory Medicine. 2020; 144: 799 805.

[31] Chen H, Guo J, Wang C, Luo F, Yu X, Zhang W, et al. Clinical characteristics and intrauterine vertical transmission potential of Covid-19 infection in nine pregnant women: a retrospective review of medical records. The Lancet. 2020; 395: 809-815.

[32] Liang H, Acharya G. Novel corona virus disease (Covid-19) in pregnancy: what clinical recommendations to follow? Acta Obstetricia et Gynecologica Scandinavica. 2020; 99: 439-442.

[33] Cavalcante MB, Sarno M, da Silva ACB, Araujo Júnior E, Barini R. Is there any possible link between Covid-19 and human infertility? The Journal of Maternal-Fetal \& Neonatal Medicine. 2020; 1-2.

[34] Vaiarelli A, Bulletti C, Cimadomo D, Borini A, Alviggi C, Ajossa S, et al. Covid-19 and ART: the view of the Italian society of fertility and sterility and reproductive medicine. Reproductive BioMedicine Online. 2020; 40: 755-759. 